\title{
Evaluación de las estrategias de vacunación contra la tos convulsa mediante un modelo matemático de transmisión de la enfermedad
}

\author{
Assessment of pertussis vaccination strategies using a \\ mathematical model of disease transmission
}

\author{
Lic. Pablo Pesco ${ }^{a}$, Dra. Paula Bergero ${ }^{a}$, Dr. Gabriel Fabricius ${ }^{a}$ y Dra. Daniela Hozbor ${ }^{b}$
}

\section{RESUMEN}

La tos convulsa o coqueluche es una enfermedad respiratoria inmunoprevenibleque ha resurgido enlasúltimasdécadas.Lamayormorbimortalidad se registra en los lactantes, aunque también se detectan casos en adolescentes y adultos. La situación epidemiológica de la enfermedad ha obligado a revisar e implementar nuevas estrategias para mejorar su control.

Sin embargo, muchas de estas estrategias aún no cuentan con un sustento experimental que permita su universalización. En este contexto, los modelos matemáticos de transmisión de enfermedades resultan herramientas útiles en la toma de decisiones.

En este trabajo se evaluó, mediante un modelo matemático para coqueluche, el impacto que tendrían distintas medidas de control en la población más vulnerable ( 0 a 1 año). En particular, se analizó el impacto de la inclusión de un refuerzo a los 11 años, el efecto de la mejora en las coberturas de las dosis primarias y la disminución del retraso en la aplicación de estas. También se estimó el efecto de la vacunación a embarazadas.

Los resultados muestran que la inclusión de un refuerzo a los 11 años disminuye un 3\% la incidencia en los menores de 1 año. Por su parte, la aplicación de las dosis primarias a tiempo calendario (sin retrasos) la reduce un $16 \%$. Al aumentar la cobertura del $80 \%$ al $95 \%$, la incidencia en la población vulnerable se reduce significativamente (38\%). Cuando el porcentaje de las embarazadas inmunizadas alcanza el $50 \%$, la reducción de los casos más graves en los infantes superaría el 43\% (0 - 2 meses).

Palabras clave: tos convulsa, Bordetella pertussis, vacunaantipertussis, inmunización, refuerzo, modelo.

http:/ /dx.doi.org/10.5546/aap.2013.377

\section{INTRODUCCIÓN}

La tos convulsa (o coqueluche) es una enfermedad respiratoria aguda altamente contagiosa causada por Bordetella pertussis. La mayoría de los casos graves afectan a menores de 1 año no inmunizados o con esquemas de inmunización incompletos que no confieren protección. ${ }^{1-3}$ Las principales complicaciones son la hospitalización, la bronconeumonía, las convulsiones, la encefalopatía aguda, el daño cerebral permanente y la muerte. ${ }^{4}$

La enfermedad se transmite de persona a persona. El paciente es más infeccioso durante las tres primeras semanas de iniciados los síntomas. Este lapso se acorta significativamente (5 días) con el tratamiento antibiótico apropiado.

Para controlar la enfermedad, se emplean vacunas celulares o acelulares. La utilización de las distintas vacunas depende, entre otros factores, de la edad de la población. Así, el uso de las vacunas celulares no está recomendado en los niños mayores de 7 años. En relación con las vacunas acelulares, existen formulaciones para la población pediátrica y formulaciones para la población adolescente y adulta, que se diferencian fundamentalmente en la dosis de inmunógenos incluidos. El uso masivo de vacunas redujo de manera significativa la morbimortalidad de la enfermedad y produjo un cambio en su epidemiología, aumentando el peso relativo de casos en los lactantes y en la población adolescente y adulta en lugar de en los niños. ${ }^{5-7}$ En la era de la vacunación, los adolescentes y los adultos actuarían como la principal fuente de contagio para los niños pequeños. ${ }^{8}$ En la era prevacunación, dada la alta circulación de la bacteria en la población, los adultos adquirían con mayor frecuencia refuerzos naturales por las recurrentes exposiciones. Las madres así inmunizadas transferían la 
protección a sus hijos, haciendo que la proporción de los lactantes que enfermaban gravemente fuera menor. La mayoría de los casos graves ocurrían en los niños de entre 3 y 6 años.

En los últimos años, en varios países se ha registrado un aumento sostenido de casos. ${ }^{5,9-11}$ En la Argentina, a partir de 2002, se comenzó a detectar un incremento que sigue hasta hoy. Como ejemplo, durante 2012, se notificaron al Sistema Nacional de Vigilancia (SIVILA) 8670 casos con sospecha de coqueluche; de estos, 6911 eran menores de 1 año. La mayoría de los casos correspondieron a las provincias más pobladas del país (Buenos Aires, Córdoba y Santa Fe). Del total, 1117 casos se confirmaron en el laboratorio. Durante 2010, se registraron 4981 casos, 828 confirmados en el laboratorio. Una vez más, la mayoría de los casos eran menores de 1 año (4217). La gran proporción de casos en lactantes no fue inesperada, ya que la tos convulsa es más grave en este grupo de edad.

Diversas causas podrían contribuir al aumento de casos detectados: fortalecimiento de la vigilancia, implementación de nuevas metodologías diagnósticas, relativa baja eficacia de las vacunas, corta duración de la inmunidad generada por las vacunas y adaptabilidad del agente causal a la inmunidad que confieren las vacunas. ${ }^{5,12,13}$

Con independencia de las causas, el aumento significativo en el número de casos ha obligado a los sistemas de salud de los distintos países a revisar e implementar nuevas estrategias para mejorar su control, en particular en la población más vulnerable: los niños menores de un año. Estas estrategias comprenden la vacunación en capullo, la vacunación del personal de salud en contacto con niños, la inmunización durante el embarazo y el agregado de un refuerzo a los adolescentes. ${ }^{14-16}$ Dada la implementación reciente de algunas de ellas, y la consecuente falta de datos, no es posible todavía universalizar ninguna estrategia. En este contexto, los modelos matemáticos que simulan la transmisión de la coqueluche pueden servir de apoyo a la toma de decisiones. ${ }^{17-20}$

En este trabajo presentamos las predicciones de nuestro modelo sobre el impacto en la incidencia de la población más vulnerable de algunas de las estrategias que se aplican en nuestro país, y el efecto que tendría la mejora de las coberturas de las dosis primarias y el cumplimiento sin retrasos del Calendario Nacional de Vacunación (CNV).

\section{POBLACIÓN Y MÉTODOS}

Los datos referidos a la edad de aplicación de las dosis de vacuna incluidos en este estudio son retrospectivos y provienen del Centro Vacunatorio del Hospital Elena de la Serna Montes de Oca, ubicado en la zona céntrica de La Plata, Provincia de Buenos Aires. Se analizó el período comprendido entre enero de 2005 y mayo de 2012, que incluyó 29845 registros de vacunación antipertussis de niños de entre 0 y 12 meses. Se excluyeron los niños mayores de 12 meses, los residentes fuera de la provincia y aquellos cuyos datos de vacunación o de edad fueran incompletos o confusos.

En la Argentina, el esquema de vacunación contempla tres dosis primarias a los 2,4 y 6 meses de edad (DPT3), una dosis a los 18 meses y refuerzos en el ingreso escolar y a los 11 años. ${ }^{21}$ Excepto la dosis de los 11 años (Tdap11, por sus siglas en inglés), en la que se emplea una formulación acelular, en las otras dosis se utilizan vacunas celulares combinadas con otros inmunógenos.

La cobertura de vacunación en el primer año de vida en la Argentina, según la OMS, varió entre el $87 \%$ y el $94 \%$ en los años 2006 a 2011, con el mínimo en 2009. ${ }^{22}$ Cuando se desagregan las coberturas de vacunación de DPT3 por departamento, se observan en alguno de ellos valores por debajo del $80 \% .^{23}$

\section{Modelo}

En este estudio utilizamos un modelo matemático determinista desarrollado por nuestro grupo, que simula la transmisión de la coqueluche. ${ }^{24}$ El modelo incluye una estructuración de la población de acuerdo con la edad, el estado inmunitario y la contagiosidad de los individuos. En el modelo se considera una población estratificada en 9 clases epidemiológicas y 30 grupos etarios. La dinámica de la propagación de la coqueluche se simula mediante la transferencia de individuos entre las clases a diferentes tasas específicas, como se muestra en la Figura 1.

En este modelo, los individuos al nacer (si no han recibido anticuerpos de la madre) se encuentran en la clase denominada susceptible $S$, donde permanecen a menos que: a) se infecten y se vuelvan infecciosos como consecuencia del contacto con una persona infectada y pasen a la clase infecciosa sintomática $\mathrm{I}_{1} \mathrm{o}, \mathrm{b}$ ) adquieran inmunidad parcial con la primera dosis de vacuna y entren en la clase $\mathrm{P}_{\mathrm{AI}}^{1}$ (del inglés partial acquired 
immunity). Cuando los individuos reciben dosis sucesivas de vacunas (indicado en la Figura 1 con líneas de puntos), pueden atravesar las clases de inmunidad creciente $\mathrm{y}$, eventualmente, llegar a la clase $\mathrm{C}_{\mathrm{AI}}$ (del inglés complete acquired immunity), que indica inmunidad completa adquirida por vacunación. Hemos considerado que solo una fracción (VE, del inglés vaccine efficacy) de los individuos que reciben una dosis dada de vacuna pasan a la clase de inmunidad siguiente, ya que es sabido que la vacuna antipertussis no tiene una eficacia del $100 \%$ (véase el Anexo en la versión electrónica). Los individuos de las clases $\mathrm{P}_{\mathrm{AI}}^{1} \mathrm{y}$ $\mathrm{P}_{\mathrm{AI}}^{2}$ pueden infectarse (y entrar en las clases $\mathrm{I}_{2} \mathrm{O}$ $\mathrm{I}_{3}$ respectivamente) y desarrollar enfermedad, pero con una sintomatología menor $y$, por ende, son menos contagiosos. La tasa a la cual los individuos susceptibles (o con inmunidad parcial) en un dado grupo etario $i$ se infectan se denomina fuerza de infección $\lambda_{i}$. El cómputo de $\lambda_{i}$ tiene en cuenta los patrones de contacto entre individuos de distintos grupos etarios, las fracciones de individuos infectados en cada uno de ellos y su respectiva contagiosidad.

A partir de la resolución de las ecuaciones diferenciales que describen la dinámica del modelo, puede calcularse la incidencia específica por edad en cada grupo etario $i$ : $\operatorname{Inc}_{1 \mathrm{i}}=\lambda_{\mathrm{i}} \mathrm{S}_{\mathrm{i}}$ (casos con sintomatología completa), $\operatorname{Inc}_{2 \mathrm{i}}=\lambda_{\mathrm{i}} \mathrm{P}_{\mathrm{AIi}}^{1}$ (casos con sintomatología parcial) donde $S_{i}$ y $\mathrm{P}_{\mathrm{AIi}}^{1}$ son las poblaciones de las clases $\mathrm{S}$ y $\mathrm{P}_{\mathrm{AI}}^{1}$ respectivamente para individuos en el grupo etario $i$.

En particular nos centralizaremos en la incidencia $\left(\mathrm{Inc}_{1}+\mathrm{Inc}_{2}\right)$ en el rango etario de $0 \mathrm{a}$ 1 año.

\section{Parametrización}

El modelo requiere parámetros que contienen información de las características de la enfermedad y su transmisión, y de la vacunación. Algunos de ellos son de difícil determinación, como la duración de la inmunidad, aún no consensuada entre los expertos. Otros parámetros no son homogéneos en la población, como las coberturas de vacunación y los patrones de contacto específicos por edades. Para contemplar esta variabilidad, hemos considerado diferentes escenarios donde se combinan los valores de los parámetros de modo de cubrir un amplio rango de situaciones posibles. Este proceder nos permite explorar la sensibilidad de los resultados ante cambios en los parámetros y distinguir así

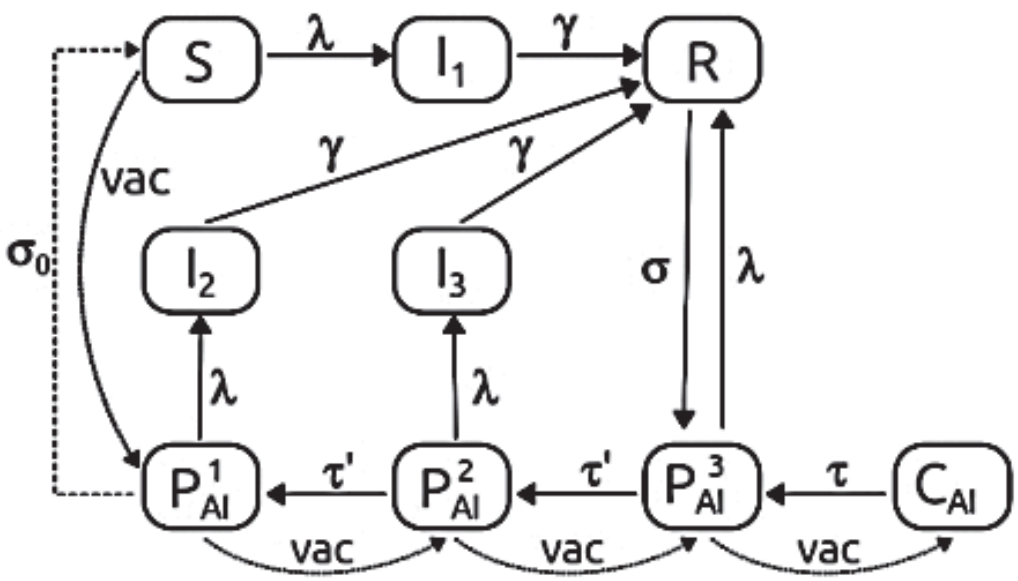

En el modelo, los individuos nacen en la clase $\mathrm{S}$ (si no recibieron anticuerpos de la madre), entonces se infectan y pasan a la clase $\mathrm{I}_{1}$ o son parcialmente inmunizados con la primera dosis de vacuna y entran en la clase $\mathrm{P}_{\mathrm{AI}}^{1}$. Con las dosis sucesivas de vacuna, los individuos atraviesan las clases $\mathrm{P}_{\mathrm{AI}}^{1} \rightarrow \mathrm{P}_{\mathrm{AI}}^{2} \rightarrow \mathrm{P}_{\mathrm{AI}}^{3} \rightarrow \mathrm{C}_{\mathrm{AI}}$, teniendo en esta última inmunidad completa. Los individuos en las clases $\mathrm{P}_{\mathrm{AI}}^{1}$ y $\mathrm{P}_{\mathrm{AI}}^{2}$ pueden infectarse entrando en las clases $\mathrm{I}_{2}$ o $\mathrm{I}_{3}$ respectivamente. La infección se extingue en un tiempo $1 / \gamma=21$ días, tras el cual los individuos en $\mathrm{I}_{1}, \mathrm{I}_{2}$ o $\mathrm{I}_{3}$ se recuperan y entran en la clase R. En el modelo se considera que la inmunidad, adquirida por infección o vacunación, no dura toda la vida sino que se pierde gradualmente. Los individuos en cualquiera de las clases con inmunidad parcial o completa recorren las clases $\mathrm{P}_{\mathrm{AI}}$ descendiendo en el nivel de inmunidad adquirida a las tasas indicadas $\left(\sigma, \tau, \tau^{\prime}\right)$. En un plazo muy largo $\left(1 / \sigma_{0}\right)$, pueden volverse totalmente susceptibles $\left(P_{A I}^{1} \rightarrow S\right)$. 
como robustos aquellos que no dependen del escenario particular considerado. En este trabajo presentamos los resultados obtenidos utilizando el escenario CP1A-MDI descrito anteriormente. ${ }^{24}$ En el escenario CP1A-MDI se parametrizan los patrones de contacto entre los individuos a partir de valores de fuerzas de infección estimados de datos epidemiológicos de la era pre-vacunación, y se supone una duración media de la inmunidad de 15 años para la adquirida por infección y de 6 para la adquirida mediante vacunación.

\section{RESULTADOS}

\section{Efecto del retraso de la vacunación primaria}

La vacunación realizada fuera del tiempo recomendado en el CNV aumenta el riesgo de enfermedad. En la Argentina, si bien han mejorado las coberturas de vacunación, aún se detectan demoras en la aplicación de las dosis recomendadas. ${ }^{25,26}$

A partir de los registros analizados hemos detectado que, en una alta proporción, las dosis DPT3 no se aplican en el tiempo indicado en el CNV (Figura 2). El 49\% de los niños recibieron la primera dosis de vacuna con al menos 1 semana de retraso, extendiéndose en algunos hasta más allá de 40 días. Respecto de la segunda y la tercera dosis, se registraron retrasos de al menos una semana en $67 \%$ y $74 \%$ de los niños, respectivamente.

Para evaluar el impacto del retraso en la vacunación sobre la incidencia de coqueluche en el grupo de 0 a 1 año, se compara la incidencia en los niños de esta edad para dos coberturas de las dosis primarias $(80 \%$ y $95 \%$ ) y para dos perfiles de vacunación (A, sin retrasos y $\mathrm{B}$, con retrasos) (Figura 3). Los resultados muestran que cuando no existen retrasos en la aplicación de las dosis, la incidencia en los menores de 1 año disminuye respecto de la calculada considerando los retrasos. Así, por ejemplo, la aplicación de las dosis primarias sin retrasos y con una cobertura del 95\% lleva a una reducción de la incidencia en los menores de 1 año del 16\%.

\section{Efecto de una mejora en las coberturas de la vacunación primaria}

Dado que la cobertura de DPT3 es heterogénea a lo largo del país, hemos realizado los cálculos considerando dos de las coberturas informadas: $95 \%$ y $80 \%$. El incremento de la cobertura desde el $80 \%$ al $95 \%$ para las dosis primarias lleva a una reducción de la incidencia en los más vulnerables del 38\% cuando las dosis se aplican a término (Figura 3, panel $A$ ) y del $36 \%$ en caso de retraso (Figura 3, panel B). Los resultados refieren a la

FIGURA 2. Distribución de la edad de aplicación de las tres dosis primarias de vacuna antipertussis

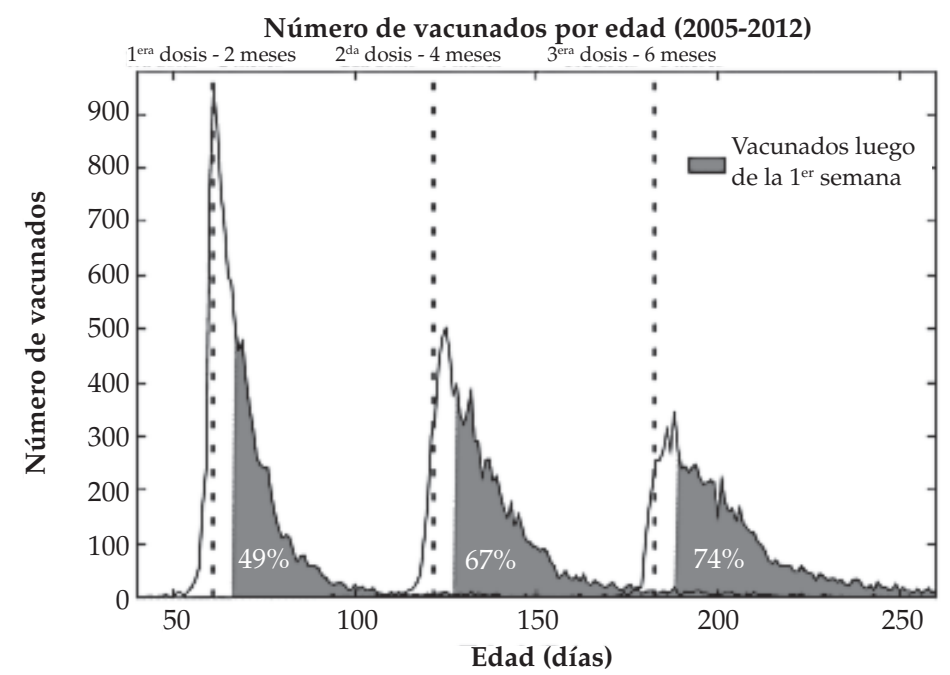

Los datos provienen del Centro Vacunal Hospital Elena de la Serna de Montes de Oca y corresponden al promedio del período 2005-2012. Los perfiles anuales fueron construidos con 3468, 3677, 2433, 4418, 4687, 4449, 5219 y 1494 registros respectivamente y presentan escasa variación respecto del promedio. En líneas verticales de puntos se señalan las edades recomendadas según el CNV para la aplicación de cada dosis primaria antipertussis. Se indican, además, los porcentajes de vacunados con más de una semana de retraso para cada dosis. 
suma de las incidencias denominadas Inc (casos $_{1}$

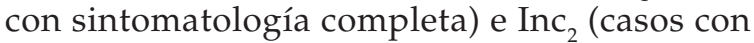
infección moderada). En los casos en que la cobertura es del 95\%, la Inc constituye un $43 \%$ de la suma Inc ${ }_{1}+\mathrm{Inc}_{2}$. Cuando la cobertura es del $80 \%$, la Inc 1 representa un $53 \%$ de esta suma, por lo cual una mejora en la cobertura implicaría la ventaja adicional de reducir los casos más graves.

\section{Efecto de la introducción de la dosis de los 11 años}

El objetivo principal del refuerzo Tdap11 es reducir la incidencia de coqueluche en los adolescentes, pero también en los menores de 1 año. Si bien la inmunización de los adolescentes parece implicar una reducción de la fuente de contagio para los menores de un año, no hay evidencia que apoye esta hipótesis.

En nuestro modelo, la incorporación de Tdap11 produjo una reducción significativa de la incidencia en los adolescentes (37\%). Sin embargo, la incidencia en la población más vulnerable (Figura 3) presentó una disminución menor del 3\%. Para los cálculos, se consideró que la cobertura de la dosis Tdap11 es del 72\% (dato de 2011) cuando las coberturas de las dosis primarias se consideraron del $80 \%$, y del $85 \%$ cuando la cobertura de las dosis primarias fue del 95\%.

Asimismo, una mejora del $85 \%$ al $95 \%$ en la cobertura de la dosis de los 18 meses llevó a una reducción del 10\% para el grupo etario de 18 meses a 3 años, pero solo redujo 1\% la incidencia de la enfermedad en los menores de 1 año.

\section{Efecto de la inmunización de las madres}

Nuestro modelo permite considerar el efecto de la transferencia de inmunidad de las madres a los recién nacidos si se incluye una clase adicional de individuos que adquieren anticuerpos maternos. ${ }^{23}$ Así, una fracción de las madres que están en la clase $\mathrm{R}$-suponiendo que los anticuerpos duran 2,5 años después de una infección- da lugar a individuos protegidos durante sus dos primeros meses. Los individuos de esta clase, al infectarse, desarrollarían una enfermedad con sintomatología menor $\left(\mathrm{I}_{2}\right)$. Al considerar tal efecto en este estudio, el modelo registra una reducción en $\mathrm{Inc}_{1}$ de alrededor del $6 \%$.

Con esta modificación del modelo es posible estimar, en forma aproximada, el efecto en los

Figura 3. Comparación de las incidencias de coqueluche en el rango etario de 0 a 1 año cuando las dosis se aplican a término (A) y con los retrasos observados en la Figura 2 (B)

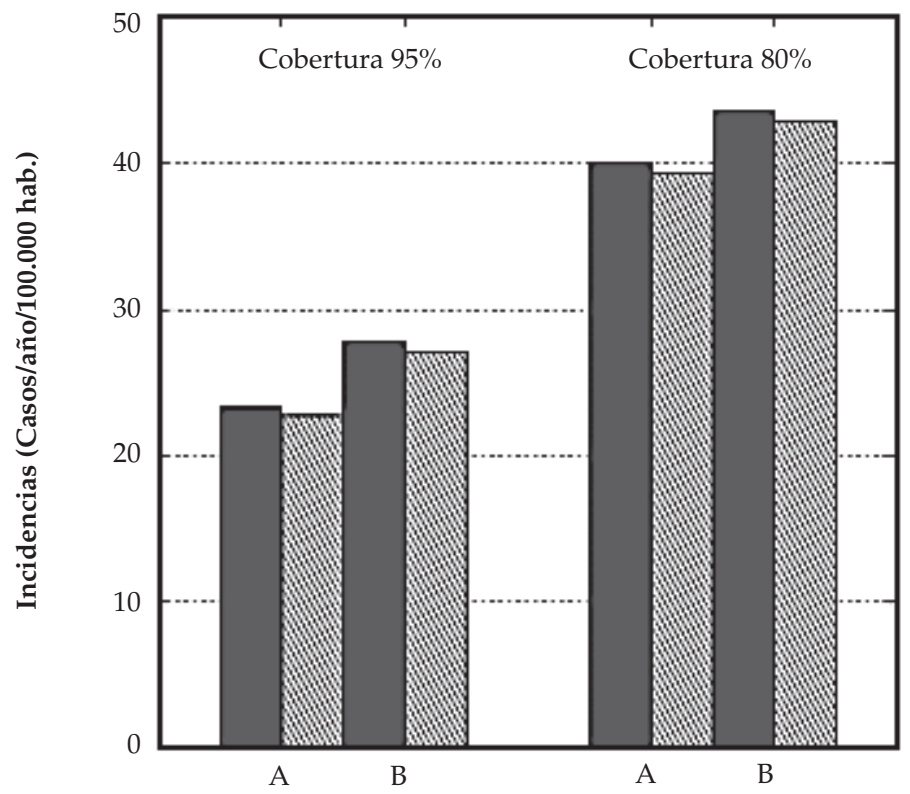

Se comparan los resultados para coberturas de $95 \%$ y $80 \%$. Las barras rayadas muestran el efecto adicional de introducir un refuerzo a los 11 años. 
lactantes de la trasmisión de inmunidad debido a la vacunación de las madres. Si consideramos, por ejemplo, que el $50 \%$ de las madres transmiten la inmunidad a su bebé, encontramos que se reduce en un $43 \%$ la aparición de casos graves en menores de 2 meses (Inc. 1). Este resultado señala la importancia de esta estrategia que protege a la población que no puede ser inmunizada mediante vacunas. Para realizar esta estimación, hemos utilizado coberturas de $95 \%$ para las tres primeras dosis y un perfil con retrasos.

\section{DISCUSIÓN}

La situación epidemiológica de la tos convulsa en la Argentina y en otros países obliga a la revisión de las estrategias de control contra la enfermedad. Los análisis aquí realizados muestran los beneficios de alcanzar coberturas del 95\% para las tres dosis primarias y de aplicarlas en el tiempo recomendado en el CNV, evitando los retrasos cuando sea posible. Es importante recordar que el perfil de retrasos utilizados se corresponde a la población de la zona céntrica de La Plata; sin embargo, es esperable que en las áreas suburbanas los retrasos sean aún mayores.

Dayan y cols. ${ }^{26}$ analizaron los motivos que conducen a los retrasos en la vacunación y notaron que algunos de ellos -por ejemplo la falta de vacunas o el desconocimiento del calendario- pueden ser prevenibles con las medidas adecuadas, mientras que otros -como las enfermedades o convalecencias en el momento de la aplicación- no son susceptibles de mejoras.

Respecto del refuerzo de los 18 meses, una mejora en la cobertura solo reduce $1 \%$ la incidencia de la enfermedad en los menores de 1 año de edad. Asimismo, la incorporación de la vacunación en la población de 11 años lleva a una pequeña reducción en la incidencia del grupo de 0 a 1 año. Esta predicción está de acuerdo con lo informado por otros autores, que utilizan diferentes modelos y parametrizaciones. ${ }^{17,27-30}$ Esto podría deberse, al menos en parte, al peso relativamente bajo que tienen los adolescentes como fuente de infección de los lactantes. ${ }^{30-33}$ Los adultos (en particular los padres) parecen ser la principal fuente de infección de los lactantes. ${ }^{34}$

Con respecto a la inmunización de las madres, en este trabajo hemos considerado solo el efecto de transmisión de anticuerpos a los recién nacidos, sin considerar explícitamente que estas madres inmunizadas dejarían de actuar como fuente de infección. La reducción estimada sobre la incidencia sería esperable que resultara aún mayor si las madres fueran inmunizadas con buenas coberturas (superiores al 50\% considerado en este trabajo).

Si bien resulta evidente la ventaja de comparar la eficacia de las medidas de control contra una enfermedad mediante un modelo matemático como el aquí utilizado, no se pretende predecir los valores absolutos de la incidencia, ya que estos valores pueden variar con la parametrización utilizada (ver el Anexo). Sin embargo, el análisis de sensibilidad realizado demuestra que el modelo reproduce de manera fidedigna el peso relativo de los distintos efectos estudiados y predice qué medidas resultarían más eficaces.

\section{CONCLUSIONES}

Según nuestro modelo, la mejora en las coberturas de las dosis primarias por encima del $95 \%$, cumpliendo sin retrasos el esquema de vacunación del Calendario Nacional, tendría un impacto significativo en la reducción de la incidencia de la tos convulsa en la población menor de 1 año (38\%). La vacunación a las embarazadas se presenta como una estrategia destacable, ya que considerando solo el efecto en la protección mediada por la transmisión de anticuerpos, la inmunización del 50\% de las madres reduce un $43 \%$ el número de casos más graves en los menores de 2 meses.

La estrategia de menor impacto sobre los infantes parece ser el refuerzo a los 11 años, que solo conduce a una leve reducción -menor del $3 \%-$ en la incidencia del grupo vulnerable.

Los ajustes en las medidas de control vigentes, que tendrían un impacto claro en la incidencia de la tos convulsa en los lactantes, deben acompañarse de nuevos estudios sobre su epidemiología y sus causas, y del diseño de nuevas estrategias que permitan el control más eficiente de esta enfermedad, hoy considerada un problema para la salud pública..

\section{Agradecimientos}

Agradecemos al Dr. Mario Arrúa su desinteresada colaboración en la provisión de parte de los datos utilizados en este estudio. Este trabajo fue financiado por la Agencia Nacional de Promoción Científica y Tecnológica (ANPCyT) y el Consejo Nacional de Investigaciones Científicas y Técnicas (CONICET). Pablo Pesco es becario del CONICET, Paula Bergero y Gabriel Fabricius son miembros de la carrera científica del CONICET y Daniela Hozbor es miembro de la carrera científica de CICBA. 


\section{BIBLIOGRAFÍA}

1. WHO. Pertussis: immunization surveillance, assessment and monitoring. [Consulta: 6 de jun. 2008]. Disponible en http://www.who.int/immunization_monitoring/ diseases/pertussis/en/index.html.

2. Dauer CC. Reported whooping cough morbidity and mortality in the United States. Pub Health Rep 1943;58:66177.

3. MattooS,CherryJD.Molecularpathogenesis, epidemiology, and clinical manifestations of respiratory infections due to Bordetella pertussis and other Bordetella subspecies. Clin Microbiol Rev 2005;18:326-82.

4. CDC. Pertussis complications. [Consulta: 27 de dic. 2012]. Disponible en http://www.cdc.gov/pertussis/about/ complications.html.

5. Cherry J. Epidemic pertussis in 2012--the resurgence of a vaccine-preventable disease. N Engl J Med 2012;367:785-7.

6. Anon. Pertussis-United States, 1997-2000. Morbidity and Mortality Weekly Report 2002;51:73-76.

7. Riva Posee CA, Miceli INP. Evolución de la coqueluche en la Argentina a finales del siglo XX. Medicina 2005;65:7-16.

8. Wiley KE, Zuo Y, Macartney KK, McIntyre PB. Sources of pertussis infection in young infants: A review of key evidence informing targeting of the cocoon strategy. Vaccine 2012 in press doi: 10.1016/j.vaccine.2012.11.052.

9. de Melker HE, Coyin-van Spaendonck, Rumke HC, van Wijngaarden JK, et al. Pertussis in The Netherlands: an outbreak despite high levels of immunization with wholecell vaccine. Emer Infect Dis 1997;3:175-8.

10. Hozbor D, Mooi F, Flores D, Weltman G, et al. Pertussis epidemiology in Argentina: trends over 2004-2007. J Infect 2009;59:225-31.

11. Base de datos del Ministerio de Salud de Argentina. [Consulta: 27 de dic. 2012]. Disponible en: http://msal. gov.ar/htm/site/pdf/epidemiologia/boletinsemanal/ BoletinIntegradoDeVigilanciaVersionVF_SE34.pdf.

12. Wendelboe AM, Van Rie A, Salmaso S, Englund J. Duration of immunity against pertussis after natural infection or vaccination. Pediatr Infect Dis Jour 2005;24:S58-61.

13. Bart MJ, van Gent M, van der Heide HG, Boekhorst J, et al. Comparative genomics of prevaccination and modern Bordetella pertussis strains. BMC Genomics 2010;11:627.

14. CDC. Updated recommendations for use of tetanus toxoid, reduced diphtheria toxoid and acellular pertussis vaccine (Tdap) in pregnant women and persons who have or anticipate having close contact with an infant aged $<12$ months. Advisory Committee on Immunization Practices (ACIP), 2011. Morbidity and Mortality Weekly Reports 2011;60:1424-26.

15. Forsyth KD, Wirsing von Konig, Tan T, Caro J, et al. Prevention of pertussis: recommendations derived from the second Global Pertussis Initiative roundtable meeting. Vaccine 2007;25:2634-42.

16. Brooks DA, Clover R. Pertussis Infection in the United States: Role for Vaccination of Adolescents and Adults. J Am Board Fam Med 2006;19:603-11.

17. Hethcote HW. Simulations of pertussis epidemiology in the United States: effects of adult booster vaccinations. Mathemat Biosc 1999;158:47-73.

18. van Boven M, de Melker HE, Schellekens JFP, Kretzschmar
M. Amodelbased evaluation of the 1996-7 pertussis epidemic in the Netherlands. Epidemiol Infect 2001;127:73-85.

19. Rohani P, Zhong X, King AA. Contact network structure explains the changing epidemiology of pertussis. Science 2010;330:982-5.

20. Lavine JS, King AA, Bjornstad ON. Natural immune boosting in pertussis dynamics and the potential for longterm vaccine failure. PNAS 2011;108:7259-64.

21. Ministerio de salud (Argentina). Calendario nacional de vacunación. [Consulta: 27 de dic. 2012]. Disponible en http://www.msal.gov.ar/index.php/component/ content/article / 46/184-calendario-nacional-devacunacion-2012.

22. OMS.WHO-UNICEF estimates of immunization coverage: Argentina. [Consulta: 27 de dic. 2012]. Disponible en: http://apps.who.int/immunization_monitoring/en/ globalsummary/timeseries/TSWUcoverageByCountry. $\mathrm{cfm}$ ? country=ARG.

23. Base de datos del Ministerio de Salud (Argentina). [Consulta: 27 de dic. 2012]. Disponible en: http://msal. gov.ar/htm/site/pdf/AnexoVII_PAI-03-09.pdf.

24. Fabricius G, Bergero PE, Ormazabal ME, Maltz AL, et al. Modelling pertussis transmission to evaluate the effectiveness of an adolescent booster in Argentina. Epidemiol Infect 2013;141(4):718-34.

25. Hethcote HW. An age-structured model for pertussis transmission. Mathem Biosc 1997;145:89-136.

26. Dayan GH, Shaw KM, Baughman AL, Orlellana LC, et al. Assessment of delay in age-appropriate vaccination using survival analysis. Am J Epidemiol 2006;163:561-70.

27. Gentile A, Bakir J, Firpo V, Caruso M, et al. Esquemas atrasados en la vacunación y oportunidades perdidas de vacunación en niños de hasta 24 meses: estudio multicéntrico. Arch Argent Pediatr 2011;103(3):219-25.

28. Quinn HE, McIntyre PB. The impact of the adolescent pertussis immunization, 2004-2009: lessons from Australia. Bull World Health Organization 2011;89:666-74.

29. Skoff TH, Cohn AC, Clark TA, Messonnier NE, et al. EarlyImpact of the USTdap vaccination program on pertussis trends. Arch Pediat Adol Med 2012;166:344-9.

30. Lavine JS, Bjornstad ON, de Blasio BF, Storsaeter J. Shortlived immunity against pertussis, age-specific routes of transmission, and the utility of a teenage booster vaccine. Vaccine 2012;30:544-51.

31. Rozenbaum MH, De Vries R, LE HH, Postma MJ. Modelling the impact of extended vaccination strategies on the epidemiology of pertussis. Epidemiol Infect 2011. doi:10.1017/S0950268811002354.

32. Jardine A, Conaty SJ, Lowbridge C, Staff M, et al. Who gives pertussis to infants? Source of infection for laboratory confirmed cases less than 12 months of age during an epidemic, Sydney, 2009. Comm Dis Intel 2010;34:116-21.

33. Bisgard KM, Pascual FB, Ehresmann KR, Miller CA, et al. Infant pertussis: who was the source? Ped Infect Dis J 2004;23:985-9.

34. Wendelboe AM, Njamkepo E, Bourillon A, Floret DD, et al. Transmission of Bordetella pertussis to young infants. Pediat Infect Dis J 2007;26:293-9.

35. AndersonR,May R. InfectiousDiseases of Humans: Dynamics and Control. Oxford: Oxford UniversityPress; 1991. 


\section{Anexo. Parámetros utilizados en el modelo}

En este trabajo consideramos que la población es constante en el tiempo, y los nacimientos y muertes se producen siguiendo una mortalidad de tipo $I_{,}^{35}$ que consiste en suponer que todos los individuos viven exactamente hasta la misma edad, que hemos tomado en 75 años. Hemos considerado que la infección dura un tiempo medio de 21 días. Para la eficacia de cada dosis de vacuna tomamos VE= 0,9 como en la referencia 25. Esto implica que entre 1 y 2 años de aplicada la tercera dosis de vacuna, nuestro modelo predice que entre el $86 \%$ y el $95 \%$ estarían protegidos de contraer una forma grave de tos convulsa y entre un $63 \%$ y $81 \%$ estarían protegidos de una enfermedad moderada. En cuanto a las coberturas de las distintas dosis, en este trabajo nos referimos a dos casos: las de $95 \%$ y $80 \%$. Cuando consideramos la cobertura del 95\%, suponemos que un 95\% de la población recibe las dosis correspondientes a los 2, 4, 6 meses y 6 años, pero que una fracción menor (85\%) recibe la dosis de los 18 meses. Para la cobertura del $80 \%$, usamos el mismo criterio y suponemos que una fracción del $70 \%$ recibe la dosis de los 18 meses.

En nuestro modelo consideramos que los individuos en las clases $\mathrm{I}_{2} \mathrm{e}_{3}$ poseen menor contagiosidad que los individuos en la clase $\mathrm{I}_{1}$. Este aspecto es tenido en cuenta a través de los factores ${ }_{1} \mathrm{y}_{2}$ que, por ser menores que 1 , relativizan la contribución a la fuerza de infección de los infectados con menor contagiosidad.

$\lambda_{i}=\sum_{j} \beta_{i j} I_{j}^{*} ; I_{j}^{*}=I_{1 j}+\rho_{1} I_{2 j}+\rho_{2} I_{3 j}$

Hemos tomado $_{1}=0,5 \mathrm{y}_{2}=0,25$ como en la referencia 25 .

Parámetros de contacto infectivo y de duración de la inmunidad para el escenario CP1A-MDI

TABla 1. Tasas de contacto, $\beta_{i j}$ (en 1/año), entre individuos del grupo etario $i$ (fila) y el grupo etario $j$ (columna) para el escenario CP1A. Se omiten algunos elementos ya que la matriz es simétrica.

\begin{tabular}{|c|c|c|c|c|c|c|c|c|c|}
\hline Edad & $0-4 \mathrm{~m}$ & $4 \mathrm{~m}-1 \mathrm{a}$ & $1-3 a$ & $3-5^{a}$ & $5-10 a$ & $10-15 a$ & $15-35 a$ & $35-55 a$ & $55-75 a$ \\
\hline $0-4 \mathrm{~m}$ & 0,02 & 0,02 & 0,02 & 0,02 & 0,02 & 0,02 & 0,12 & 0,12 & 0,12 \\
\hline $4 m-1 a$ & & 0,12 & 0,12 & 0,12 & 0,12 & 0,12 & 0,23 & 0,23 & 0,23 \\
\hline $1-3 a$ & & & 0,23 & 0,23 & 0,23 & 0,23 & 0,23 & 0,23 & 0,23 \\
\hline $3-5 a$ & & & & 0,73 & 0,73 & 0,23 & 0,23 & 0,23 & 0,23 \\
\hline $5-10 a$ & & & & & 2,47 & 0,95 & 0,23 & 0,23 & 0,23 \\
\hline $10-15 a$ & & & & & & 0,95 & 0,54 & 0,16 & 0,0 \\
\hline $15-35 a$ & & & & & & & 0,54 & 0,16 & 0,0 \\
\hline $35-55 a$ & & & & & & & & 0,16 & 0,0 \\
\hline $55-75 a$ & & & & & & & & & 0,0 \\
\hline
\end{tabular}

\section{Duración de la inmunidad contra pertussis}

TABLA 2. Parámetros para la duración media de la inmunidad (MDI)

\begin{tabular}{cccccc}
\hline$t_{N}$ & $t_{v}$ & $1 / \sigma$ & $1 / \tau$ & $1 / \tau^{\prime}$ & $1 / \sigma_{0}$ \\
15 & 6 & 11 & 2 & 2 & 100 \\
\hline
\end{tabular}

$\mathrm{t}_{\mathrm{N}} \mathrm{y} \mathrm{t}_{\mathrm{V}}$ son la duración de la inmunidad adquirida naturalmente (a través de la infección) o artificialmente (a través de la vacuna), respectivamente. Las tasas $\sigma, \tau$ y $\tau^{\prime}$ se eligen de manera de obtener $t_{\mathrm{N}}=1 / \sigma+2 / \tau^{\prime}, \mathrm{t}_{\mathrm{v}}=1 / \tau+2 / \tau^{\prime}$. El ritmo al cual los individuos inmunizados se vuelven totalmente susceptibles es controlado por la tasa, $\sigma_{0}$. Todos los valores están en años. 


\section{ANÁLISIS DE SENSIBILIDAD}

En ref. [24] hemos estudiado la transmisión de pertussis utilizando distintas parametrizaciones que representan diferentes escenarios epidemiológicos. Todos estos escenarios reproducen las características conocidas de la epidemiología de pertussis y son por lo tanto"posibles". En la Tabla 3 mostramos como se modifican los principales resultados presentados en este trabajo (para el escenario
CP1A-MDI) cuando se utiliza el modelo con parametrizaciones correspondientes a otros escenarios epidemiológicos. Se observa que si bien los valores de las incidencias son diferentes entre los distintos escenarios todas las conclusiones obtenidas respecto a las estrategias vacunales analizadas se mantienen para todos los escenarios considerados y son, por lo tanto, robustas.

\section{Incidencia calculada para distintos escenarios y estrategias}

TABLA 3. Incidencias totales (Inc $1+\operatorname{Inc} 2$ ) en los menores de 1 año para distintos escenarios considerados y distintas estrategias.

\begin{tabular}{lccccccc}
\hline Escenario & $\begin{array}{c}\text { COB 95-B } \\
\mathbf{( 1 )}\end{array}$ & COB 95-A & $\begin{array}{c}\text { Cambio porcentual } \\
\text { respecto a (1) }\end{array}$ & $\begin{array}{c}\text { COB } \\
\text { 95-B +11a }\end{array}$ & $\begin{array}{c}\text { Cambio porcentual } \\
\text { respecto a (1) }\end{array}$ & $\begin{array}{c}\text { COB } \\
\mathbf{8 0 - B}\end{array}$ & $\begin{array}{c}\text { Cambio porcentual } \\
\text { respecto a (1) }\end{array}$ \\
\hline CP1A-LDI & $\mathbf{2 5 , 5}$ & 21,3 & $-16,2$ & 24,5 & $-3,7$ & 41,1 & 61,3 \\
CP1A-MDI & $\mathbf{2 7 , 5}$ & 23,3 & $-15,4$ & 27,2 & $-1,2$ & 43,6 & 58,4 \\
CP1A-SDI & $\mathbf{3 5 , 0}$ & 30,0 & $-14,5$ & 34,4 & $-1,8$ & 52,2 & 48,9 \\
CP1B-LDI & $\mathbf{3 3 , 0}$ & 27,4 & $-17,2$ & 32,7 & $-1,0$ & 49,5 & 49,8 \\
CP1B-MDI & $\mathbf{3 5 , 1}$ & 29,1 & $-17,1$ & 34,8 & $-0,9$ & 52,3 & 48,7 \\
CP1B-SDI & $\mathbf{4 3 , 6}$ & 36,8 & $-15,6$ & 43,2 & $-1,0$ & 63,6 & 45,9 \\
CP2-LDI & $\mathbf{2 1 , 6}$ & 19,1 & $-11,5$ & 20,8 & $-3,5$ & 35,2 & 63,2 \\
CP2-MDI & $\mathbf{2 8 , 2}$ & 25,1 & $-10,8$ & 27,3 & $-2,9$ & 42,7 & 51,8 \\
CP2-SDI & $\mathbf{4 6 , 4}$ & 42,1 & $-9,2$ & 45,6 & $-1,7$ & 69,8 & 50,5 \\
Promedio & $\mathbf{3 2 , 9}$ & 28,3 & $-14,1$ & 32,3 & $-2,0$ & 50,0 & 53,2 \\
DCM & $\mathbf{8 , 2}$ & 7,4 & 2,9 & 8,3 & 1,1 & 11,0 & 6,2 \\
\hline
\end{tabular}

La columna en negrita representa el caso tomado como referencia para calcular los porcentajes de cambio para cada estrategia (COB 95-B= cobertura del 95\% en las serie primaria antipertussis con perfil de retrasos de la Figura 2, COB 95-A= cobertura del $95 \%$ en las serie primaria antipertussis sin retrasos, COB $80-\mathrm{B}=$ cobertura del $80 \%$ en las serie primaria antipertussis con perfil de retrasos de la Figura 2, COB 95-B $\neg+$ Vac 11a= cobertura del 95\% en las serie primaria antipertussis con perfil de retrasos de la Figura 2 más aplicación de vacuna de 11 años con cobertura de $85 \%$ y sin retraso). Se muestran además el promedio y la desviación cuadrática media (DCM) para cada situación.

Mayores detalles sobre los parámetros considerados en el modelo y sobre el análisis de sensibilidad realizado pueden solicitarse a los autores a través del correo electrónico. 